A.E. Salvi

G.P. Metelli

A. Bosco

A. Berizzi

S.A. Hacking

A. Cantalamessa

\section{Spontaneous bilateral Achilles tendon rupture in a patient treated with oral levofloxacin}

Received: 16 January 2007

Accepted: 20 March 2007

Published online: 8 June 2007

A.E. Salvi (西) • G.P. Metelli

Orthopaedics and Traumatology

Department

Mellino Mellini Hospital Trust

Civil Hospital of Iseo

I-25049 Iseo (BS), Italy

E-mail: andrea@ orthopaedics.com

A. Bosco

Accident and Emergency Department

Mellino Mellini Hospital Trust

Civil Hospital of Iseo

Iseo (BS), Italy

A. Berizzi

Orthopaedics and Traumatology

Department

University of Padua

Padua, Italy

S.A. Hacking

Department of Orthopaedics

JTN Wong Labs for Mineralized Tissue

Research

McGill University

Montreal (Quebec), Canada

A. Cantalamessa

Department of Internal Medicine

Mellino Mellini Hospital Trust

Civil Hospital of Iseo

Iseo (BS), Italy

\begin{abstract}
A case of bilateral rupture of the Achilles tendon in a patient treated with levofloxacin for cystitis is reported. A 76-year-old woman suddenly developed painful ankles one day after levofloxacin treatment. Drug therapy was switched to amoxicillin/clavulanate on the fourth day. Sonography revealed a serious condition of tendinosis with complete bilateral full-thickness rupture on day 6 . Tendons were both repaired in the same surgical session. Pathological anatomy of the specimens reported fatty tissue lobules with panniculitis and histiocytosis. Ankles were immobilized postoperatively with a plaster cast. Achilles tendon rupture may occur as an adverse side effect of short-term use of levofloxacin, a fluoroquinolone antibiotic. This adverse effect is a rare and poorly understood complication of this antibiotic therapy. A review of the literature is provided.
\end{abstract}

Key words Fluoroquinolone • Levofloxacin • Antibiotic • Achilles tendon $\cdot$ Tendon rupture $\cdot$

Tendinosis $•$ Cystitis

\section{Introduction}

Fluoroquinolones are effective antibiotics for the treatment of infections of the upper and lower respiratory tract, urinary tract, skin [1] and eye [2]. Fluoroquinolones are often chosen for their excellent gastrointestinal absorption, superior tissue penetration and broad-spectrum activity [3,4]. Reported adverse effects include nausea, headache, diarrhea, giddiness, abdominal discomfort, 
vomiting, agitation, sleep disturbance, rash and pruritis [5]. Other rare but reported side effect are Achilles tendinitis and tendon rupture [3,6-15] especially in patients 60 years and older [2]. We describe the case of a woman who suffered complete and sudden rupture of both Achilles tendons induced by levofloxacin, an antibiotic of the fluoroquinolones group.

\section{Case report}

A 76-year-old woman, currently being treated for hypertension with enalapril who had a deep venous thrombosis four years prior, required antibiotic therapy for cystitis. In November 2006, the patient was prescribed oral levofloxacin (500 mg once daily), each morning, for five days. After just a single dose of this antibiotic, she reported pain in the ankles and difficulty walking. Before the fourth dose of levofloxacin, her family doctor changed the treatment to amoxicillin/clavulanate (1000 mg twice a day). After six days of pharmacological therapy, while the patient attempted to stand up from her sofa, she heard a "crack" sound from both ankles (more intensely from the left ankle) following which she fell to the floor and was unable to rise again. She presented to the Accident and Emergency Department of the Civil Hospital of Iseo (Brescia, Italy) and was admitted to the Orthopaedics and Traumatology Department.

Clinical examination failed to reveal any sign of hematoma on either Achilles tendons (Fig. 1), however the patient demonstrated a positive Thompson sign on both calf ten-



Fig. 1 Photograph of the ankles of a 76-year-old woman who suffered bilateral Achilles tendon rupture (prone position). No sign of hematoma is visible. Arrows, cutaneous trace left by the examiner's finger, like a "fovea sign" corresponding to the tendon lesions


Fig. 2a, b Complete rupture of the Achilles tendons. a Left tendon. As discovered using sonography, there was remarkable retraction of the rupture ends (arrow). b Right tendon. As discovered using sonography, there was little diastasis between the rupture ends

dons. On the following day, sonography and color Doppler sonography of both Achilles tendons were performed. Sonography revealed a serious condition of tendinosis, rich in myxoid degeneration, with complete bilateral full-thickness rupture. Sonography also revealed the presence of abundant hypoechoic sheets put internally the lesion, pointing out a previous subtotal lesion, recently damaged again after trauma, involving full-thickness the tendons. Color Doppler sonography did not reveal any present thrombosis. More importantly, sonography did not report any inflammatory swelling of the surrounding tissues.

Surgical intervention occurred the next day. The intraoperative findings showed complete rupture at the midportion of the Achilles tendons. The left tendon presented a larger gap (Fig. 2a) compared to the right tendon (Fig. 2b). Histological examination of samples retrieved from both tendons, performed by the Department of Pathological 


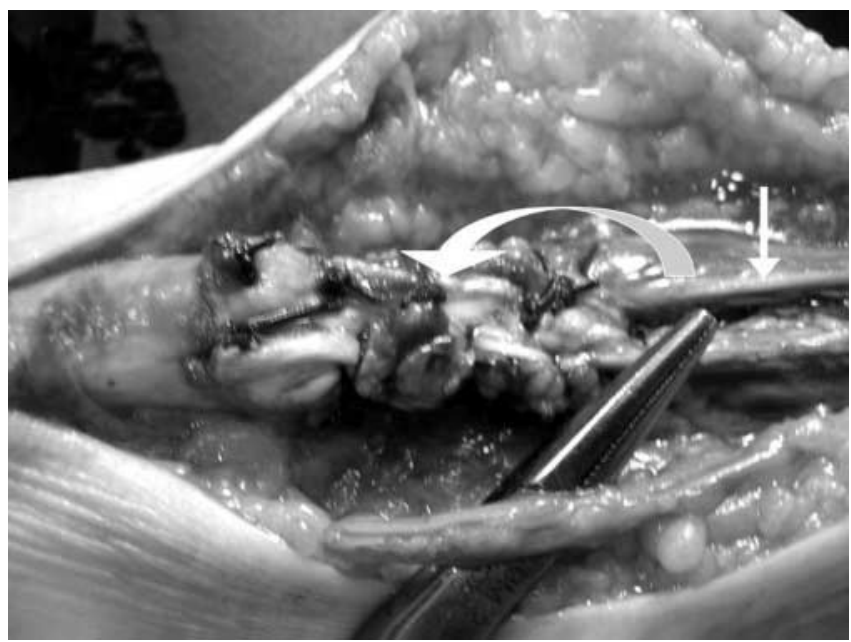

Fig. 3 The Achilles tendon ends were sutured and a patch of proximal end tendon (straight arrow), overturned $180^{\circ}$ (curved arrow), was sutured on the distal end. Fibrin glue was injected into the sutured ends. The sural nerve is visible

Anatomy of Chiari (Brescia, Italy), reported fatty tissue lobules with panniculitis and histiocytosis. A fragment of muscular tissue attached to one tendon specimen showed partial necrosis and the tendon sheath possessed fibrin and chronic inflammation.

An 'end to end' tendon suture was performed bilaterally, which was augmented with a tendon patch (rotated $180^{\circ}$ ) harvested from the proximal end of the tendon (Fig. 3 ) to add collagen to the repair site and to enhance biomechanical stability [16]. Finally fibrin glue was injected into the repair site. The operated limbs were immobilized with an above-knee plaster cast with the ankle in maximal plantar flexion for 30 days, following which the cast was replaced with a $90^{\circ}$ ankle flexion below-knee plaster cast for a further 30 days. As of January 2007 the cast has been removed and the patient has been ambulatory with progressive weight-bearing, suggesting a good outcome.

\section{Discussion}

There are many proposed mechanisms to explain Achilles tendinitis and rupture following fluoroquinolone therapy. Known risk factors and diseases that cause fragility of the tendons include advanced age (60 years and older), systemic lupus erythematosus, gout, chronic renal failure, rheumatic fever, hyperparathyroidism, hyperlipidemia type 2, syphilis, gonorrhea, tumors, rheumatoid arthritis [3, 11, 17-21] and multiple sclerosis (due to chronic corticosteroid therapy) [22]. Hypercholesterolemia associated with hypothyroidism may also contribute to delayed healing and ultimate failure of the degenerated Achilles tendon [23]. In this case, our patient was treated for hypertension but was not taking corticosteroids. Obesity may also predispose to Achilles tendon rupture by both being a marker of sedentary lifestyle that leads to tendon atrophy and increasing periodic strain on the tendon [24]. The heavy mechanical workload of the Achilles tendon (i.e. during weight-bearing activities like walking and running) [11] is also cited as another predisposing factor [7], making the Achilles tendon more vulnerable than other tendons [11].

The relationship between fluoroquinolones (norfloxacin) and Achilles tendinitis was first described by Bailey et al. [25] in 1983. The first described cases of fluoroquinolone-induced Achilles tendon rupture were reported by McEwan and Davey [26] in 1988 regarding ciprofloxacin, followed by Perrot et al. [27] and by Franck et al. [28] in 1991 regarding pefloxacin. According to Karch and Lasagna's algorithm for causality [29, 30] in order to establish a causative connection between the undesirable outcome of a medicine and the suspected medicine, we show that our case report fulfils the appropriate requirement: (a) a reasonable time has passed between taking the medicine and the happening of the event (the sudden onset of tendinopathy after a single dose of a fluoroquinolone suggests a direct toxic effect on collagen fibers) [31], (b) the reaction due to the use of levofloxacin (i.e. a fluoroquinolone) is well known and reported in literature, (c) other possible causes (e.g. traumatism, rheumatoid arthritis, systemic lupus erythematosus, use of corticosteroids, and tendon fragility due to advanced age) were excluded, and (d) symptoms got better when levofloxacin was stopped. Nevertheless, in fluoroquinoloneinduced tendinopathy, the tendon can rupture even after these antibiotics are no longer administered [1]. This may explain why in this case both Achilles tendons ruptured during amoxicillin/clavulanate therapy.

A review of the literature conducted by Khaliq and Zhanel [32] illustrated that the first intervention in case of pain includes discontinuation of the implicated fluoroquinolone. Moreover, these authors suggested that the fluoroquinolones most commonly implicated are pefloxacin (37\% of the drug-induced tendon injuries) and ciprofloxacin $(25.5 \%$ of the drug-induced tendon injuries) and that patients with a mean age of 59 years are more susceptible to experience tendon injuries when receiving fluoroquinolones. Therefore, the case we describe is concordant with this review of the literature.

Quinolones have an important affinity for connective tissues [33]. There is a preference for the Achilles tendon, but also shoulder (supraspinatus tendon and long head of biceps), hand (extensor pollicis longus) and epicondylar tendon involvement has been reported [4, 13, 20, 34-38]. 
Jorgensen et al. [39] proposed that tendon rupture could be the result of a vasculitic phenomenon leading to ischemia. These histological changes due to the cytotoxic effect of these antibiotics on tenocytes could result in a decreased tensile capacity of the tendon and rupture during normal activities [40]. According to Khaliq and Zharel [32], the diagnosis of Achilles tendons rupture is made by physical examination plus sonography and a color Doppler sonography. Cellular death due to the overproduction of free oxygen radicals during fluoroquinolone administration has been reported [2]. Nevertheless, the specific pathophysiology of fluoroquinolone-induced tendinitis and tendon rupture is poorly understood [8]. The absence of hematoma and swelling in both the ruptured Achilles tendons of this case supports the hypothesis that tendon failure resulted from physiological degeneration of the tendons exacerbated by levofloxacin administration. Therefore, elderly patients who are administered levofloxacin should be closely monitored for symptoms of tendonitis [41].

\section{References}

1. Lipsky BA, Baker CA (1999) Fluoroquinolone toxicity profiles: a review focusing on newer agents. Clin Infect Dis 28:352-364

2. Pouzaud F, Rat P, Cambourieu C et al (2002) Tenotoxic potential of fluoroquinolones in the choice of surgical antibiotic prophylaxis in ophthalmology. $\mathrm{J}$ Fr Ophthalmol 25:921-926

3. Zabraniecki L, Negrier I, Vergne P et al (1996) Fluoroquinolone induced tendinopathy: report of 6 cases. J Rheumatol 23:516-520

4. - (2002) Levaquin. In: Physicians' desk reference, 5th edn. Medical Economics, Montvale, pp 2537-2543

5. Wolfson JS, Hooper DC (1989) Fluoroquinolone antimicrobial agents. Clin Microbiol Rev 2:378-424

6. Lewis JR, Gums JG (1999) Levofloxacin-induced bilateral Achilles tendonitis. Ann Pharmacother 33:792-795

7. Harrel RM (1999) Fluoroquinoloneinduced tendinopathy: what do we know? South Med J 92:622-625

8. Kowatari K, Nakashima K, Ono A et al (2004) Levofloxacin-induced bilateral Achilles tendon rupture: a case report and review of the literature. J Orthop Sci 9:186-190

9. Dekens-Konter JA, Knol A, Olsson S et al (1994) Tendinitis of the Achilles tendon caused by pefloxacin and other fluoroquinolone derivatives. Ned Tijdschr Geneeskd 138:528-531

10. Movin T, Gad A, Guntner P et al (1997) Pathology of the Achilles tendon in association with ciprofloxacin treatment. Foot Ankle Int 18:297-299
11. Ribard P, Audisio F, Kahn MF et al (1992) Seven Achilles tendinitis including 3 complicated by rupture during fluoroquinolone therapy. J Rheumatol 19:1479-1481

12. West MB, Gow P (1998) Ciprofloxacin, bilateral Achilles tendonitis and unilateral tendon rupture-a case report. N Z Med J 111:18-19

13. Pierfitte C, Royer RJ (1996) Tendon disorders with fluoroquinolones. Therapie 51:419-420

14. Simonin MA, Gegout-Pottie P, Minn A et al (2000) Pefloxacin-induced achilles tendon toxicity in rodents: biochemical changes in proteoglycan synthesis and oxidative damage to collagen. Antimicrob Agents Chemother 44:867-872

15. Kashida Y, Kato M (1997) Characterization of fluoroquinolone-induced Achilles tendon toxicity in rats: comparison of toxicities of 10 fluoroquinolones and effects of anti-inflammatory compounds. Antimicrob Agents Chemother 41:2389-2393

16. Zell RA, Santoro VM (2000) Augmented repair of acute Achilles tendon ruptures. Foot Ankle Int 21:469-474

17. Donck JB, Segaert MF, Vanrenterghem YF (1994) Fluoroquinolones and Achilles tendinopathy in renal transplant recipients. Transplantation 58:736-737

18. Meyboom RH, Olsson S, Knol A et al (1994) Achilles tendinitis induced by pefloxacin and other fluoroquinolone derivatives. Pharmacoepidemiol Drug Saf 3:185-189

19. Price AE, Evanski PM, Waugh TR (1986) Bilateral simultaneous achilles tendon ruptures. A case report and review of the literature. Clin Orthop Relat Res 213:249-250
20. Royer RJ, Pierfitte C, Netter P (1994) Features of tendon disorders with fluoroquinolones. Therapie 49:75-76

21. Smaill GB (1961) Bilateral rupture of the Achilles tendons. BMJ 1:1657-1658

22. Fadda M, Piras S, Manunta A, Zirattu G (2006) A rare case of bilateral and simultaneous Achilles tendon rupture after levofloxacin therapy. Chir Piede 30:37-40

23. Rao SK, Navadgi BC, Vasdev A (2005) Bilateral spontaneous rupture of Achilles tendons: a case report. J Orthop Surg (Hong Kong) 13:178-180

24. Seeger JD, Pharm D, West WA et al (2006) Achilles tendon rupture and its association with fluoroquinolone antibiotics and other potential risk factors in a managed care population. Pharmacoepidemiol Drug Saf 15:784-792

25. Bailey RR, Kirk JA, Peddie BA (1983) Norfloxacin-induced rheumatic disease. N Z Med J 96:590

26. McEwan SR, Davey PG (1988) Ciprofloxacin and tenosynovitis. Lancet 2:900

27. Perrot S, Ziza JM, De Bourran-Cauet G et al (1991) A new complication related to quinolones: rupture of Achilles tendon. Presse Med 20:1234

28. Franck JL, Bouteiller G, Chagnaud P et al (1991) Achilles tendon rupture in 2 adults treated with pefloxacin, one of the cases with bilateral involvement. Rev Rhum Mal Osteoartic 58:904

29. Karch FE, Lasagna L (1977) Toward the operational identification of adverse drug reactions. Clin Pharmacol Ther 21:247-254 
30. Lado Lado FL, Rodriguez Moreno C et al (2005) Partial bilateral rupture of the Achilles tendon associated to levofloxacin. An Med Interna 22:28-30

31. Van der Linden PD, Van Puijenbroek EP, Feenstra J et al (2001) Tendon disorders attributed to fluoroquinolones: a study on 42 spontaneous reports in the period 1988 to 1998. Arthritis Rheum 45:235-239

32. Khaliq Y, Zhanel GG (2003) Fluoroquinolone-associated tendinopathy: a critical review of the literature. Clin Infect Dis 36:1404-1410

33. Shakibaei M, Pfister K, Schwabe R et al (2000) Ultrastructure of Achilles tendons of rats treated with ofloxacin and fed a normal or magnesium-deficient diet. Antimicrob Agents Chemother 44:261-266
34. Casparian JM, Luchi M, Moffat RE, Hinthorn D (2000) Quinolones and tendon ruptures. South Med J 93:488-491

35. Gabutti L, Stoller R, Marti HP (1988) Fluoroquinolones as etiology of tendinopathy. Ther Umsch 55:558-561

36. Guerin B, Grateau G, Quartier G, Durand H (1996) Rupture of the long biceps tendon following ingestion of fluoroquinolone. Ann Med Interne (Paris) 147:69 (in French)

37. Levadoux M, Carli P, Gadea JF et al (1997) Repeated rupture of the extensor tendons of the hand due to fluoroquinolones. Apropos of a case. Ann Chir Main Memb Super 16:130-133 (in French)
38. Le Huec JC, Schaeverbeke T, Chauveaux D et al (1995) Epicondylitis after treatment with fluoroquinolone antibiotics. J Bone Joint Surg Br 77:293-295

39. Jorgensen C, Anaya JM, Didry $C$ et al (1991) Arthropathy with Achilles tendon involvement induced by pefloxacin. Apropos of a case. Rev Rhum Mal Osteoartic 58:623-625 (in French)

40. Maffulli N (1999) Rupture of the Achilles tendon. J Bone Joint Surg Am 81:1019-1036

41. Burkhardt O, Kohnlein T, Pap T, Welte T (2004) Recurrent tendinitis after treatment with two different fluoroquinolones. Scand J Infect Dis 36:315-316 